\title{
A New Method of Polymer Molecular Weight Determination Based on the Permeable Sphere Model
}

\author{
Lech GMACHOWSKI \\ Institute of Physical Chemistry, Polish Academy of Sciences, \\ Kasprzaka 44/52, 01-224 Warsaw, Poland
}

(Received August 15, 1985)

\begin{abstract}
It is shown that the concentration dependence of the coils-solvent permeability coefficient, experimentally determined over a wide range, may be well described by corrected Brinkman's model for the fluid flow relative to a swarm of permeable spheres of uniform permeability. A method of checking model reliability is developed. A new method of polymer molecular weight determination is elaborated on the basis of this model. According to this method, molecular weight may be calculated from the function describing concentration dependence of permeability coefficient in a semidilute regime along with a permeability coefficient value in dilute solution.
\end{abstract}

KEY WORDS Brinkman's Model / Determination of Polymer Molecular Weight / Semidilute Polymer Solution / Permeability Coefficient / Blob Radius /

\section{THE MODEL}

The macromolecular coils in a solution are represented by a swarm of porous spheres of uniform permeability. The model presented ${ }^{1,2}$ is an extension of the model for the fluid flow relative to a swarm of identical permeable spheres, due to Brinkman. ${ }^{3}$ Figure 1 shows the basic structure of the model.

A chosen coil is surrounded by a fluid envelope embedded in a porous mass representing the other coils. Inside the coil of radius $R$, the polymer material is treated as a permeable medium and the reciprocal square root of its permeability is denoted by $P$. Outside the envelope the porous mass has the permeability $k$ and the reciprocal square root of the permeability is denoted by $\lambda$. Since the coil material is a permeable medium, the solvent flows not only around but also through the coils.

Inside the coil and outside the envelope, the solvent flow is described by the Brinkman equation:

$$
\operatorname{grad} p=\frac{\mu}{k_{i}} v+\mu \nabla^{2} v
$$

where $k_{i}=1 / \lambda^{2}$ or $1 / P^{2}$ for the surrounding porous medium and for the coil, respectively, and $\mu$ is the solvent viscosity.

Between the two spherical boundaries, the solvent flow is described by the Stokes equation:

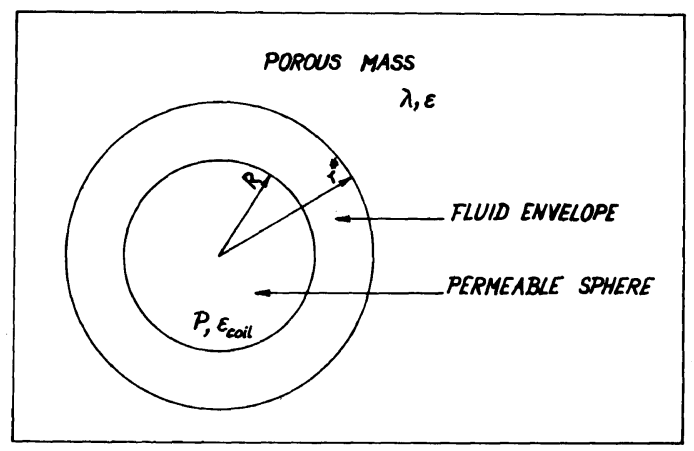

Figure 1. The Brinkman model applied to permeable spheres. 


$$
\operatorname{grad} p=\mu \nabla^{2} v
$$

The way employed in this work to attack the problem was the same as in the paper of Brinkman. ${ }^{4}$ For eq 1 and 2 , relevant stream functions were introduced. The equations, written in terms of these stream functions, were solved and the arbitrary constants appearing in the general solutions were calculated on the basis of the boundary conditions imposed. The boundary conditions at the two spherical boundaries were the continuity of pressure and the continuity of velocity and its first derivatives. In spite of the same method of solution being used, the results obtained differed from those of Brinkman. ${ }^{3}$ The difference was found due to analytical error in the work of Brinkman. The force $F_{i}$ exerted on the sphere by the fluid is given by the corrected equation:

$F_{i}=8 \pi \mu R v_{0}$

$\times \frac{\frac{2 a h}{\delta^{3}}(c h-2 a f)+a h\left(2 a n-h k_{1}\right)}{(h j-2 a m) \cdot(c h-2 a f)+(b h-2 a e) \cdot\left(2 a n-h k_{1}\right)}$

where $v_{0}$ is the sedimentation velocity, and

$$
\begin{aligned}
& a=\frac{1}{2}\left(\frac{\lambda R}{\delta}+1\right) \\
& b=-\frac{2}{3} \lambda R \\
& c=-\frac{5}{3 \delta^{2}}\left(\frac{\lambda R}{\delta}+3\right) \\
& e=\frac{T}{P^{3} R^{3}}-\frac{1}{P^{2} R^{2}}-\frac{2}{3} \\
& f=\left(\frac{10}{P^{3} R^{3}}+\frac{5}{P R}\right) T-\frac{10}{P^{2} R^{2}}-\frac{5}{3} \\
& h=1-\frac{T}{P R} \\
& j=-\frac{1}{3 \delta^{2}}\left(\frac{\lambda R}{\delta}+3\right)
\end{aligned}
$$

$$
\begin{aligned}
& k_{1}=\frac{2}{3 \lambda^{2} R^{2} \delta^{3}}\left(\frac{\lambda^{3} R^{3}}{\delta^{3}}+6 \frac{\lambda^{2} R^{2}}{\delta^{2}}+45 \frac{\lambda R}{\delta}+45\right) \\
& m=\left(\frac{2}{P^{3} R^{3}}+\frac{1}{P R}\right) T-\frac{2}{P^{2} R^{2}}-\frac{1}{3} \\
& n=\left(-\frac{10}{P^{3} R^{3}}-\frac{4}{P R}\right) T+\frac{10}{P^{2} R^{2}}+\frac{2}{3} \\
& T=\frac{\exp (P R)-\exp (-P R)}{\exp (P R)+\exp (-P R)} \\
& \delta=\frac{R}{r^{*}}
\end{aligned}
$$

The concentration $N$ of the spheres is given by

$$
N=\frac{1-\varepsilon}{\frac{4}{3} \cdot \pi R^{3}\left(1-\varepsilon_{\mathrm{coil}}\right)}
$$

where $1-\varepsilon$ and $1-\varepsilon_{\text {coil }}$ are the solute volume fractions in the solution and in the coil, respectively ( $\varepsilon$ and $\varepsilon_{\text {coil }}$ are the solution porosity and the coil porosity, respectively). Taking account of the fact that $N F_{i}$ is equal to the pressure gradient expressed by the Darcy equation:

$$
\operatorname{grad} p=\frac{\mu}{k} v_{0}
$$

one obtains the equation defining the dimensionless parameter $\lambda R$.

$$
\lambda R=\sqrt{6 \frac{1-\varepsilon}{1-\varepsilon_{\mathrm{coil}}} \cdot \frac{F_{i}}{8 \pi \mu R v_{0}}}
$$

where

$$
\lambda=1 / \sqrt{k}
$$

To complete the model, the outer radius $r^{*}$ of the fluid envelope associated with any sphere within the swarm must be determined. In this work, it is defined in such a way that the total porosity inside the outer sphere $\left(r=r^{*}\right)$ is identical with that of the original swarm. This requires that 


$$
\frac{\frac{4}{3} \pi R^{3}\left(1-\varepsilon_{\mathrm{coii}}\right)}{\frac{4}{3} \pi\left(r^{*}\right)^{3}}=1-\varepsilon
$$

which is equivalent to

$$
\delta^{3}=\left(\frac{R}{r^{*}}\right)^{3}=\frac{1-\varepsilon}{1-\varepsilon_{\text {coil }}}
$$

The $\delta^{3}$-parameter thus represents the volume fraction occupied by coils.

The model represented by eq 3-5 may be used to calculate the permeability of a swarm of permeable spheres at a given concentration, when their radius, porosity and permeability are known.

The model may also be regarded as representing an interdependence of three dimensionless parameters

$$
f\left(\lambda R, P R, \delta^{3}\right)=0
$$

\section{VERIFICATION OF THE MODEL}

The present model is valid in dilute solution considered as consisted of a swarm of individual coils. However, the applicability of the model may be extended.

In semidilute solution, the permeability is independent ${ }^{5,6}$ of molecular weight. Consider two solutions 1 and 2 and assume that the molecular weight of polymer in solution 1 , $M_{1}$ is higher than that in solution $2, M_{2}$ so that the porosity of the solution $1, \varepsilon_{1}^{*}$ at the dilute-semidilute region transition, is higher than that of solution $2, \varepsilon_{2}^{*}$. Up to a concentration corresponding to the porosity $\varepsilon_{2}^{*}$, the solution 1 has the same permeability as the solution 2. With respect to solution permeability, the entangled polymer solution is equivalent to the unentangled polymer solution 2 at its critical porosity $\varepsilon_{2}^{*}$.

In such a way, the Brinkman model, originally derived for the dilute regime, is also applicable to semidilute one.

It was stated theoretically ${ }^{5}$ and verified experimentally ${ }^{6}$ that the permeability coefficient of a given polymer solution in the semidilute regime is a power function of concentration. Hence, the $\lambda$-parameter may be described as

$$
\lambda=\text { const }_{1}(1-\varepsilon)^{n}
$$

This function determines a straight line in the $\ln \lambda v s . \ln (1-\varepsilon)$ plot and represents the inflection point of the $\ln \lambda R v s . \ln \delta^{3}$ model curve* at a $P R$-value such that

$$
\left.\frac{\mathrm{d} \ln \lambda R}{\mathrm{~d} \ln \delta^{3}}\right|_{P R=\text { const }}=n
$$

The $n$-value thus determines, on the basis of the model represented by eq 6 , the location of the inflection point, and corresponding to it the values of $\lambda R, P R$, and $\delta^{3}$, which remain unchanged along the line represented by eq 7

$$
\begin{aligned}
& \lambda R=\text { constant } \\
& P R=\text { constant } \\
& \delta^{3}=\text { constant }
\end{aligned}
$$

According to eq 7 , the $\lambda$-parameter increases with concentration of solution and the $R$-value should decrease by eq 8 . From eq 7 and 8 one gets the following proportionality

$$
R \sim(1-\varepsilon)^{-n}
$$

The same result was obtained by Brochard and de Gennes ${ }^{5}$ with respect to the blob radius. In the semidilute region eq 5,8,9, and 10 do not describe the macromolecule parameters $R, P$ and $\varepsilon_{\text {coil }}$ but the corresponding blob parameters. The blob parameters become equal to the macromolecule ones at the dilute-semidilute region transition porosity. The corresponding value of $\varepsilon^{*}$ depends on the molecular weight of dissolved polymer. The molecular weight is determined as the weight of a sphere of the radius $R$ and the density

* The detailed proof of this statement will be published elsewhere. 
$\rho_{\mathrm{s}}\left(1-\varepsilon_{\text {coil }}\right)$ relative to the weight of ${ }^{12} \mathrm{C}$ iso- tions tope of carbon

$$
M=\frac{\frac{4}{3} \pi R^{3} \rho_{\mathrm{s}}\left(1-\varepsilon_{\mathrm{coil}}\right)}{1.66 \cdot 10^{-27}}=\text { const }_{2} R^{3}\left(1-\varepsilon_{\mathrm{coil}}\right)
$$

The critical porosity $\varepsilon^{*}$ for a given molecular weight $M$ may be calculated by eq 5,7 , 8,10 , and 11 as follows

$$
\varepsilon^{*}=1-\left[\left(\frac{\lambda R}{\text { const }_{1} \delta}\right)^{3} \cdot \frac{\text { const }_{2}}{M}\right]^{1 /(3 n-1)}
$$

Then one is able to calculate the macromolecule parameters $R, P$, and $\varepsilon_{\text {coil }}$ from eq 5,710 , and 12

$$
\begin{aligned}
& R=\left[\left(\frac{\lambda R}{\text { const }_{1} \delta^{3 n}}\right)^{1 / n} \cdot \frac{\text { const }_{2}}{M}\right]^{-(n /(3 n-1))} \\
& P=P R\left[\left(\frac{\lambda R}{\text { const }_{1} \delta^{3 n}}\right)^{1 / n} \cdot \frac{\text { const }_{2}}{M}\right]^{n /(3 n-1)} \\
& \varepsilon_{\text {coil }}=1-\left[\left(\frac{\lambda R}{\text { const }_{1} \delta^{3 n}}\right)^{3} \cdot \frac{\text { const }_{2}}{M}\right]^{1 /(3 n-1)}
\end{aligned}
$$

By assuming that the values of parameters $R, P$, and $\varepsilon_{\text {coil }}$ at the point transition remain unchanged over the whole range of dilute solution, it is possible to calculate by eq $3-5$ the curve $\lambda v s .(1-\varepsilon)$, valid for a chosen $M$-value in the dilute regime.

To check the above method of calculations, sedimentation velocity data for poly ( $\alpha$-methyl styrene) (PAMS) of three different molecular weights in cyclohexane, measured by Mijnlieff and Jaspers, ${ }^{7}$ were utilized. The $\lambda$ value corresponding to a given solution concentration $c$ was calculated by the following equation

$$
\lambda=\sqrt{\frac{\left(1-\rho_{1} / \rho_{\mathrm{s}}\right) c}{\mu \mathrm{s}}}
$$

where $\rho_{1}$, and $\rho_{\mathrm{s}}$ are the solvent density and solute density, respectively, and $s$ is the sedimentation coefficient. This equation was obtained from eq 4 by the following substitu-

$$
F_{i}=\frac{4}{3} \pi R^{3}\left(\rho_{\mathrm{s}}-\rho_{1}\right)\left(1-\varepsilon_{\mathrm{coil}}\right) \frac{v_{0}}{s}
$$

where $F_{i}$ is the centrifugal force acting on the coil, allowing for the buoyancy of the surrounding solvent, and

$$
c=\rho_{\mathrm{s}}(1-\varepsilon)
$$

For higher concentrations, the $\lambda$-value and solute volume fraction had a straight line relationship on logarithmic paper. In this region, the empirical function was found in the form

$$
\lambda=6.7 \times 10^{9}(1-\varepsilon) \quad\left[\mathrm{m}^{-1}\right]
$$

The value of $n=1$ determined the coordinates of the point of inflection of the $\ln \lambda R v s . \ln \delta^{3}$ model curve. By eq 3-5 they were calculated as equal to

$$
\begin{aligned}
& \lambda R=3.88 \\
& P R=6.83 \\
& \delta^{3}=0.539
\end{aligned}
$$

The molecular weight, taking the value of PAMS density into account, was

$$
M=2.86 \times 10^{30} R^{3}\left(1-\varepsilon_{\text {coil }}\right) ;[\mathrm{R}]=m
$$

From the above equations, the value of critical porosity $\varepsilon^{*}$ and the macromolecule parameters $R, P$, and $\varepsilon_{\text {coil }}$ were calculated by eq 12 and $13-15$, respectively, for three given $M$-values. The lines calculated by eq $3-5$ on the basis of these parameters are depicted in Figure 2 together with experimental points which form an empirical straight line in the semidilute regime.

The points are quite close to the theoretical curves, especially at low concentrations. The very good agreement at low concentrations is probably due to the negligible coil contact in very dilute solutions. However, when the polymer concentration increases, the polymer coils start touching one another, and the "effective molecular weight" of particles increases 


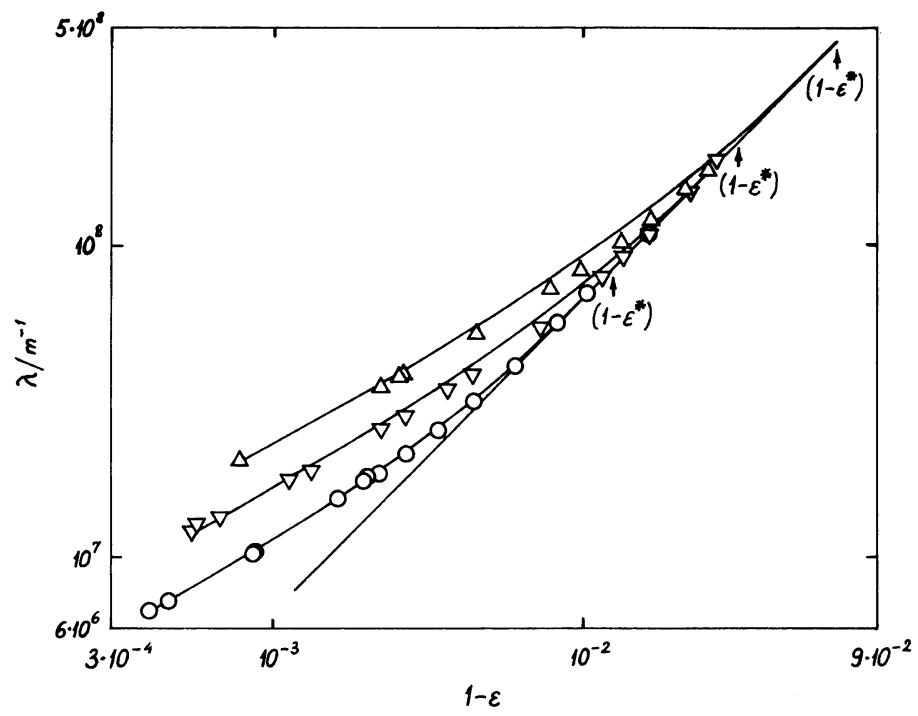

Figure 2. Plots of reciprocal square root of permeability against concentration for three PAMS samples in cyclohexane: $\triangle, M=2.34 \times 10^{5} ; \nabla, M=1 \times 10^{6} ; \bigcirc, M=6.5 \times 10^{6}$.

with concentration. As a result, experimental points lie under the respective theoretical curves and merge with the collective line at a concentration lower than that calculated from $\delta^{3}$ and $\varepsilon_{\text {coil }}$ values on the basis of eq 5 .

\section{MOLECULAR WEIGHT DETERMINATION}

The very good agreement of the theoretical curves with experimental data for very dilute solutions indicates that the present model may be used for determination of the molecular weight of a polymer investigated. The procedure for this determination is the following. The exponent $n$ in eq 7 determines the values of $\lambda R, P R$, and $\delta^{3}$ in the semidilute regime, as explained above. Then a trial-anderror method is employed. Assume the value of polymer molecular weight $M$. By eq $13-15$, the macromolecule parameters are calculated. On the basis of these parameters, eq 3-5 make it possible to determine the $\lambda$-value for a concentration $(1-\varepsilon)$ in the dilute regime. If the calculated value of $\lambda$ is equal to the measured one, then the $M$-value is assumed prop- erly and molecular weight determination is over.

In the case of PAMS-data, using the points of the lowest concentrations investigated, the $M$-values calculated were very close to their nominal values. The calculated values were $6.66 \times 10^{6}, 1.01 \times 10^{6}$, and $2.14 \times 10^{5}$, whereas the nominal values were equal to $6.5 \times 10^{6}$, $1 \times 10^{6}$, and $2.34 \times 10^{5}$, respectively. If one uses data for a higher concentration, for which polymer coils probably start touching one another, a higher $M$-value will be obtained, corresponding to an "effective radius" $R_{\text {eff }}$ of a coil. To ensure that the $M$-values determined are reliable, the limiting concentration should be defined.

It seems that volume concentration of coils has the largest influence on the effective coil radius $R_{\text {eff }}$ relative to the coil radius $R$. The volume concentration of coils in different polymer-solvent solutions is approximately the same for equal values of packing fraction of coils, defined here as the ratio of solution concentration $(1-\varepsilon)$ to the maximum of possible concentration $\left(1-\varepsilon^{*}\right)$ in the dilute regime, and denoted by $\xi$ : 


$$
\xi=\frac{1-\varepsilon}{1-\varepsilon^{*}}
$$

As a consequence of the above considerations, the following equation may be written down

$$
\frac{R_{\mathrm{eff}}}{R}=f(\xi)
$$

From eq 13, the following proportionality may be derived

$$
R \sim M^{n /(3 n-1)}
$$

which is valid for any polymer-solvent system at porosities $\varepsilon \geqslant \varepsilon^{*}$. From eq 16 and 17 one obtains

$$
\left(\frac{M_{\text {eff }}}{M}\right)^{n /(3 n-1)}=f(\xi)
$$

This equation makes it possible to compare the values of $\left(M_{\text {eff }} / M\right)$ calculated for different polymer-solvent systems for the same packing fraction of coils since the form of $f(\xi)$ is supposed to be independent of polymer-solvent system. To support this hypothesis, $M_{\text {eff }}$-values were calculated for higher concentration of PAMS in cyclohexane and the results were compared with those obtained from data ${ }^{7}$ of PAMS in toluene. In the case of PAMStoluene system, the empirical equation valid in semidilute regime was of the form:

$$
\lambda=5 \times 10^{9}(1-\varepsilon)^{0.8} \quad\left[\mathrm{~m}^{-1}\right]
$$

Unfortunately, most experimental points belonged to the semidilute regime and the rest were close to the collective line described by eq 19. In this connection, the $M_{\text {eff }}$-values calculated for points of the lowest concentration were higher than the nominal $M$-values.

The two polymer-solvent systems analyzed greatly differ because cyclohexane is a poor solvent for PAMS and measurements were performed at the theta temperature, whereas toluene is a good solvent for PAMS and this system was investigated far from theta conditions. In spite of such a difference, the points

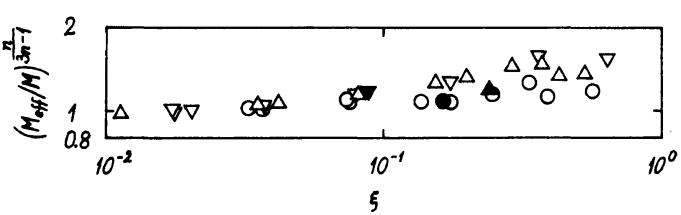

Figure 3. Plots of $\left(M_{\mathrm{eff}} / M\right)^{n /(3 n-1)}$ vs. $\xi$ for PAMS of the following molecular weights: $M=2.34 \times 10^{5}(\triangle$, in cyclohexane; $\boldsymbol{\Delta}$, in toluene); $M=1 \times 10^{6}(\nabla$, in cyclohexane; $\boldsymbol{\nabla}$, in toluene); $M=6.5 \times 10^{6}$ ( $\bigcirc$, in cyclohexane; $\boldsymbol{\odot}$, in toluene).

in Figure 3 are quite close to one another.

The points in Figure 3 form a single curve for coil packing fractions less than 0.1 . In such a region, it is thus possible to use permeability data to calculate the molecular weight of a polymer. For $\xi<0.03$ the above absolute method of molecular weight determination may be applied because $M_{\text {eff }}$ is very close to nominal molecular weight. For a small packing fraction of coils $\xi<0.1$ it will be possible to develop a relative method of polymer molecular weight determination.

\section{REMARKS}

Although the number of analyzed systems is small, the proposed method of polymer molecular weight determination may be considered experimentally verified because:

1) All polymer-solvent systems are very similar to one another with respect to sedimentation behaviour, both in the semidilute region (a power scaling law of the form of eq 7) and in very dilute region (hypothetical linear dependence of the reciprocal of sedimentation coefficient on concentration).

2) The model presented is applicable both the semidilute region and very dilute region, independent of the character of polymer-solvent system.

3) Only two regions of concentration are important in utilizing the present absolute method for determining molecular weight. 


\section{Molecular Weight Determination}

\section{REFERENCES}

1. P. M. Adler, P. M. Mills, and D. C. Quemada, AIChE J., 24, 354 (1978).

2. L. Gmachowski, presented at the 8th International Congress of Chemical Engineering, Chemical Equipment Design and Automation/CHISA/the 298th Event of the European Federation of Chemical Engineering, Prague, September 3-7, 1984.
3. H. C. Brinkman, Appl. Sci. Res., A1, 81 (1948).

4. H. C. Brinkman, Proc. Acad. Sci. Amsterdam, 50, 618 (1947).

5. F. Brochard and P. G. de Gennes, Macromolecules, 10, 1157 (1977).

6. J. Roots and B. Nyström, Polymer, 20, 148 (1979).

7. P. F. Mijnlieff and W. J. M. Jaspers, Trans. Faraday, Soc., 67, 1837 (1971) 